Received: 29.08 .2021

Revised: 21.10 .2021

Accepted: 29.10 .2021

DOI: $10.17804 / 2410-9908.2021 .5 .036-043$

\title{
MICROSTRUCTURE EVOLUTION IN A Fe-Ni-Si ALLOY DURING HEAT TREATMENT AND ELECTRON IRRADIATION
}

\author{
S. E. Danilov \\ M.N. Miheev Institute of Metal Physics, Ural Branch of the Russian Academy of Sciences, \\ 18 S. Kovalevskoy St., Ekaterinburg, 620108, Russian Federation \\ https://orcid.org/0000-0001-9815-8196 danilov@imp.uran.ru \\ *Corresponding author: E-mail: danilov@imp.uran.ru \\ Address for correspondence: 18, ul. S. Kovalevskoy, Ekaterinburg, 620990, Russian Federation \\ Tel.: +7 (343) 3783846
}

Experiments have been made on studying structural and phase changes by the residual resistivity method in a Fe-Ni-Si alloy during annealing and electron irradiation. Thermal diffusion starts above $600 \mathrm{~K}$ and ensures decomposition of the solid solution up to $700 \mathrm{~K}$; at higher temperatures it provides homogenization of the solid solution with the dissolution of intermetallic compounds in accordance with the equilibrium phase diagram. It is shown that, upon irradiation with electrons above $240 \mathrm{~K}$, vacancy clusters are formed in both the aged and quenched alloys. Dissociation of clusters occurs at temperatures ranging between 350 and $500 \mathrm{~K}$ and leads to solid solution decomposition under the influence of diffusion caused by migrating vacancies with the formation of intermetallic precipitates. Irradiation at $423 \mathrm{~K}$ causes migration of vacancies and decomposition of the solid solution. In alloys irradiated at both temperatures above $700 \mathrm{~K}$, as well as in unirradiated alloys, the precipitates dissolve in accordance with a single equilibrium dependence.

Keywords: microstructure, Fe-Ni-Si alloy, irradiation, intermetallic compounds, electrical resistance, homogenization, solid solution decomposition, diffusion.

\section{Acknowledgment}

The work was performed under the state assignment from the Ministry of Education and Science of Russia, theme Function, No. 0388-2019-0004.

\section{References}

1. Voyevodin V.N., Neklyudov I.M., Bryk V.V., Borodin O.V. Microstructural evolution and radiation stability of steels and alloys. J. Nucl. Mater., 1999, vol. 271-272, pp. 290-295. DOI: 10.1016/S0022-3115(98)00785-5.

2. Dimitrov C., Dimitrov O. Composition dependence of defect properties in electron irradiated Fe-Cr-Ni solid solutions. J. Phys. F: Metal Phys., 1984, vol. 14, pp. 793-811. DOI:10.1088/0305-4608/14/4/005.

3. Arbuzov V.L., Goshchitskii B.N., Danilov S.E., Kar'kin A.V., Perminov D.A. Effect of neutron and electron irradiation on radiation-induced separation of solid solutions in the $\mathrm{Fe}-\mathrm{Ni}$ and Fe-Ni-P ALLOYS. The Physics of Metals and Metallography, 2008. vol. 106, No. 3. pp. 266-275. DOI: 10.1134/S0031918X08090068.

4. $\quad$ Sagaradze V.V., Pavlov V.A., Alyabiev V.M., Goshchitskiy B.N., Kozlov A.V., Lapin S.S., Loguntsev Ye.N., Nalesnik V.M., Khakhalkin N.V., Shalayev V.I., Gaydukov M.G., Sergeyev G.A. The influence of intermetallic ageing during irradiation by fast neutrons on void formation in austenitic stainless steels. The Physics of Metals and Metallography, 1988, vol. 65, No. 5, pp. 128-135. 
5. Druzhkov A.P., Perminov D.A., Davletshin A.E. The effect of alloying elements on the vacancy defect evolution in electron-irradiated austenitic $\mathrm{Fe}-\mathrm{Ni}$ alloys studied by positron annihilation. J. Nucl. Mat., 2009, 384 (1), pp. 56-60. DOI: 10.1016/j.jnucmat.2008.10.002.

6. Druzhkov A.P., Arbuzov V.L., and Danilov S.E. The effect of deuterium and tritium on formation and annealing of vacancy-type defects in deformed nickel. Physica Status Solidi (a) Applications and Materials Science, 2008, vol. 205, iss. 7, pp. 1546-1551. DOI: 10.1002/pssa.200723494.

7. Couderchon G., Porteseil J.L. Some properties of nickel-rich commercial Fe-Ni alloys. In: G. Beranger et al., eds. The Iron-Nickel alloys, Lavoisier Publishing, 1996, pp. 29-58.

8. $\quad$ Arbuzov V.L., Danilov S.E., Druzhkov A.P., Perminov D.A. The influence of plastic deformation and radiation defects on the structural and phase transformations of N36 and N36T2 austenitic alloys. The Physics of Metals and Metallography, 2004, vol. 98, No. 5, pp. 500-505.

9. Takeda Shuzo, Iwama Yoshiroh, Sakakura Akira. On the Equilibrium Diagram of Fe-Ni-Si System and the Ternary Compound y-Phase Appearing in This System (Fundamental Research of Constant Permeability Alloys, 2nd Report). Journal of the Japan Institute of Metals and Materials, 1960, vol. 24, iss. 8, pp. 534-538. DOI: 10.2320/jinstmet1952.24.8_534.

10. Alisova S.P., Budberg P.B., Ageev N.V. Diagrammy sostoyaniya metallicheskikh system [State diagrams of metal systems]. Moscow, Lyubertsy, VINITI Publ., 1971, iss. 15, 263 p. (In Russian). 
Подана в журнал: 29.08.2021

УДК 539.12.04:621.78: 669.15.24

DOI: $10.17804 / 2410-9908.2021 .5 .036-043$

\title{
ЭВОЛЮЦИЯ МИКРОСТРУКТУРЫ В СПЛАВЕ Fe-Ni-Si ПРИ ТЕРМООБРАБОТКЕ И ОБЛУЧЕНИИ ЭЛЕКТРОНАМИ
}

\author{
С. Е. Данилов \\ Федеральное государственное бюджетное учреждение науки \\ Институт физики металлов им. М.Н. Михеева Уральского отделения Российской академии наук, \\ д. 18, ул. С. Ковалевской, Екатеринбург, Российская Федераџия \\ iD https://orcid.org/0000-0001-9815-8196 danilov@imp.uran.ru \\ *Ответственный автор. Электронная почта: danilov@imp.uran.ru \\ Адрес для переписки: ул. С. Ковалевской, 18, Екатеринбург, Российская Федерация \\ Тел.: +7 (343) 378-38-46
}

Проведены эксперименты по исследованию структурно-фазовых изменений методом остаточного электросопротивления в сплаве $\mathrm{Fe}-\mathrm{Ni}-\mathrm{Si}$ при отжигах и электронном облучении. Термическая диффузия начинается при температурах выше $600 \mathrm{~K}$ и обеспечивает распад твердого раствора до $700 \mathrm{~K}$, а при более высоких температурах - гомогенизацию твердого раствора с растворением интерметаллидов в соответствии с равновесной фазовой диаграммой.

Показано, что при облучении электронами выше 240 К происходит образование вакансионных кластеров как в состаренном, так и в закаленном сплаве. Диссоциация кластеров происходит в температурном интервале 350-500 К и приводит к распаду твердого раствора под действием диффузии обусловленной мигрирующими вакансиями с образованием интерметаллидных выделений. Облучение при температуре 423 К приводит к миграции вакансий и распаду твердого раствора. В сплавах, облученных при обеих температурах выше $700 \mathrm{~K}$, как и в необлученных сплавах происходит растворение выделений в соответствии с единой равновесной зависимостью.

Ключевые слова: микроструктура, сплав $\mathrm{Fe}-\mathrm{Ni}-\mathrm{Si}$, облучение, интерметаллиды, электросопротивление, гомогенизация, распад твердого раствора, диффузия.

\section{1. Введение}

В условиях интенсивного облучения в конструкционных сталях и сплавах развиваются различные радиационно-индуцированные структурно-фазовые превращения, которые в значительной степени определяют изменения свойств материалов и их радиационную повреждаемость. В сплавах с пересыщенным твердым раствором при облучениях и термообработках может происходить радиационно-ускоренный распад твердого раствора с образованием выделений другой фазы. При этом состояние матрицы приближается к термодинамически равновесному. Этот процесс непосредственно связан со свободной миграцией точечных дефектов, генерируемых облучением, которая ускоряет диффузию и позволяет получить такие состояния, обычно недостижимые при обычных термических обработках.

При миграции неравновесных радиационных дефектов в сплавах между ними могут происходить реакции, приводящие к образованию комплексов радиационных дефектов с примесными атомами, вакансионных кластеров (ВК), дислокационных петель, и т. п. [1]. Кроме того, выделения второй фазы и ВК являются стоками для точечных дефектов и могут существенно уменьшить длину диффузионного пробега радиационных дефектов и изменить эффективность их влияния на структурно-фазовые превращения при облучении [1]. Fe-Ni-aустенитные сплавы, во многом аналогичные нержавеющим сталям, легированные $\mathrm{Ti}, \mathrm{Al}$ или Si образуют при 
старении дисперсную когерентную упорядоченную $\boldsymbol{\gamma}^{\prime}$ фазу типа $\mathrm{Ni}_{3} \mathrm{Me}$ [2-4], что приводит к существенному сдерживанию радиационного распухания [4].

Легирующие элементы влияют существенным образом на эволюцию как твердого раствора, так и на выделения второй фазы. Размерный фактор легирующих элементов играет решающую роль в характере их взаимодействия с точечными дефектами в твердом растворе. В никеле, аустенитных $\mathrm{Fe}-\mathrm{Cr}-\mathrm{Ni}$ сталях и $\mathrm{Fe}-\mathrm{Ni}$-сплавах подразмерные примеси (P, Si) взаимодействуют преимущественно с межузельными атомами [1, 4-6], а надразмерные примеси $(\mathrm{Ti}, \mathrm{Nb})$ - с вакансиями $[1,7,8]$. Образование стабильных комплексов вакансий или межузельных атомов с примесями служит усилению рекомбинации точечных дефектов и, следовательно, изменяет кинетику образования кластеров дефектов. Эти процессы могут оказывать существенное влияние на кинетику радиационно-стимулированной сегрегации, ползучести и порового распухания.

Цель работы - изучение влияния легирования железо-никелевого сплава кремнием на термические и радиационно-индуцированные структурно-фазовые превращения.

\section{2. Образцы и методики}

Исследования были проведены на чистом сплаве $\mathrm{Fe}-31,2$ ат. \% Ni-5,7aт. \% $\mathrm{Si}$ (Fe-Ni-Si), приготовленном вакуумным индукционным переплавом из чистых компонентов. Сплав для исследований был взят в состоянии, закаленном от $1373 \mathrm{~K}$ в воду с целью получения максимально гомогенизированного закаленного состояния (3), и в состоянии, состаренном после закалки (С) при различных температурах 823-723 К. Образцы в закаленном состоянии, исследованные с помощью рентгеноструктурного анализа на установке ДРОН-2, представляли из себя однофазный аустенит. Исследования микроструктуры проводились на электронном микроскопе JEM-200 CX при ускоряющем напряжении 160 кВ. Исследования показали, что образцы имели размер зерна 50 мкм и плотность дислокаций около $10^{7} \mathrm{~cm}^{-2}$. Облучения 5 МэВ электронами этих сплавов при различных температурах и изохронные отжиги со средней скоростью нагрева $1 \mathrm{~K} /$ мин были проведены в атмосфере чистого проточного гелия.

В качестве метода исследования было использовано измерение остаточного электросопротивления, чувствительного к процессам атомного перераспределения в твердом растворе. Остаточное электросопротивление при температуре 4,2 К измеряли стандартным четырехзондовым потенциометрическим методом с чувствительностью на уровне 0,01 нОмсм и точностью не хуже $0,05 \%$.

\section{3. Результаты и обсуждение}

На рис. 1 а представлены изменения электросопротивления при изотермическом отжиге закаленных от 1373 К образцов сплава Fe-Ni-Si при разных температурах выше $700 \mathrm{~K}$. На рис. 1 б представлены изменения электросопротивления этих сплавов при последующем изохронном отжиге совместно с результатами изохронного отжига закаленного от $1373 \mathrm{~K}$ сплава. При изотермическом отжиге при различных температурах в интервале 723-823 К происходит быстрый рост электросопротивления в пределах нескольких минут до различного уровня и при дальнейшем изотермическом отжиге электросопротивление сплавов остается практически постоянным. Поскольку в сплавах такого типа происходит термически активированное зарождение и рост интерметаллидных выделений [4], полагаем, что наблюдаемый рост электросопротивления соответствует этому процессу. Как видно, с повышением температуры изотермического отжига, уровень этого квазистационарного состояния электросопротивления уменьшается. Это связано с увеличением растворимости кремния в твердом растворе сплава Fe-Ni-Si с повышением температуры [9]. Важно, что равновесное состояние твердого раствора при выбранных температурах изотермического отжига, достигаемое за несколько минут, может быть достигнуто и при других способах отжига, например при прохождении этих температур при изохронном отжиге (рис. 1 б). Точки, соответствующие температурам 
изотермического отжига и значениям электросопротивления, получаемого при этом, обозначены черными кружками. Эти кружки лежат на равновесной зависимости.

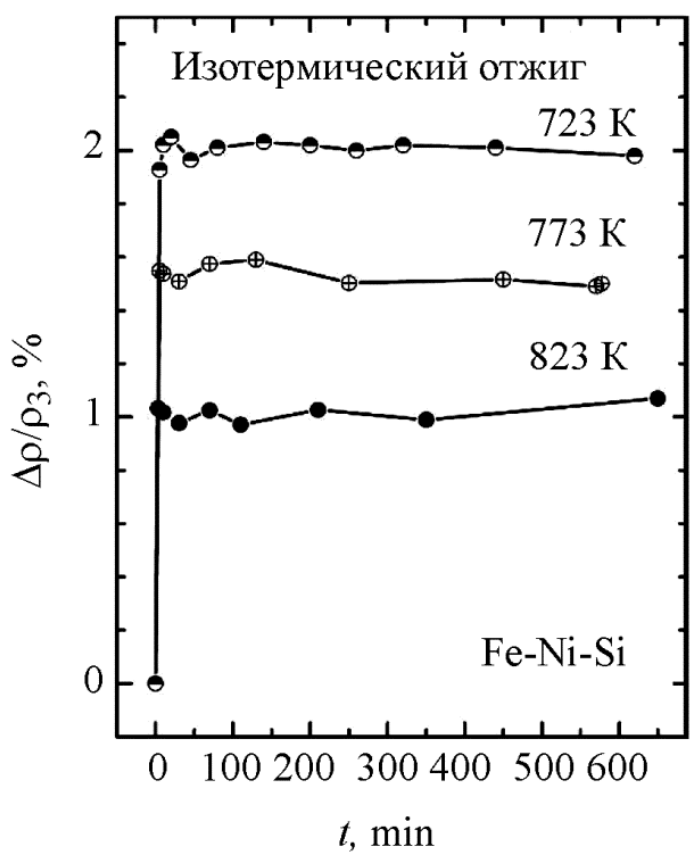

$a$

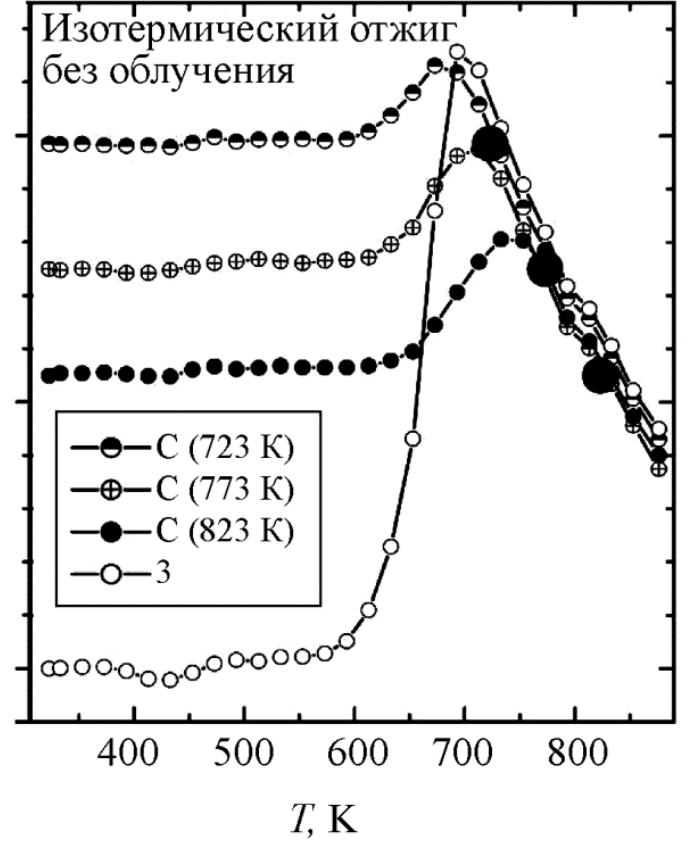

б

Рис. 1. Электросопротивление при изотермическом отжиге закаленных образцов сплавов

$\mathrm{Fe}-\mathrm{Ni}$-Si и изохронном отжиге этих сплавов в состаренном (C) состоянии совместно с изохронным отжигом сплава в закаленном (3) состояниии. В качестве исходного значения $\rho_{3}$ выбрано электросопротивление сплава, закаленного от 1373 К. $\Delta \rho=\rho-\rho_{3}$

Как видно из результатов изохронного отжига закаленного сплава, термическая диффузия становится заметной при температуре выше 600 К. При этом как в закаленном, так и в состаренных сплавах, происходит термически активированный рост интерметаллидных выделений. Выше 700 К в соответствии с равновесной растворимостью кремния [10] происходит их растворение. При температурах выше $700 \mathrm{~K}$ все зависимости сходятся в единую зависимость электросопротивления от температуры, определяемую равновесной зависимостью количества образованных интерметаллидных выделений в твердом растворе сплава $\mathrm{Fe}-\mathrm{Ni}-\mathrm{Si}$.

На рис. 2 представлены результаты изменения электросопротивления при облучении электронами при 243 и 423 К закаленного и состаренного (823 K) сплава Fe-Ni- Si, а также изменения электросопротивления при изохронном отжиге этих сплавов после облучения. При облучении наблюдается рост электросопротивления при $423 \mathrm{~K}$, а при $243 \mathrm{~K}$ роста практически нет (рис. 2 a). Считаем, что рост электросопротивления обусловлен, как и в случае термического старения, распадом твердого раствора с образованием интерметаллидных выделений. Можно сделать вывод, что при 423 К этот процесс обеспечивается подвижными вакансиями, а при 243 К, когда вакансии имеют короткие диффузионные пути этот процесс практически не обнаруживается. При 243 К вакансии только накапливаются в виде ВК.

Как было отмечено нами ранее, в сплавах на основе $\mathrm{Fe}-\mathrm{Ni}$ в районе температур 350-500 К происходит диссоциация вакансионных кластеров [3]. Наблюдаемый на рис. 26 рост электросопротивления при изохронном отжиге на сплавах $\mathrm{Fe}-\mathrm{Ni}-\mathrm{Si}$, облученных при 243 К в этом же температурном интервале, связан с образованием выделений второй фазы. При изохронном отжиге в области температур 350-500 К происходит диссоциация ВК, образованных облучением, и свободная миграция вакансий, приводящая к распаду твердого раствора с образованием интерметаллидов. 


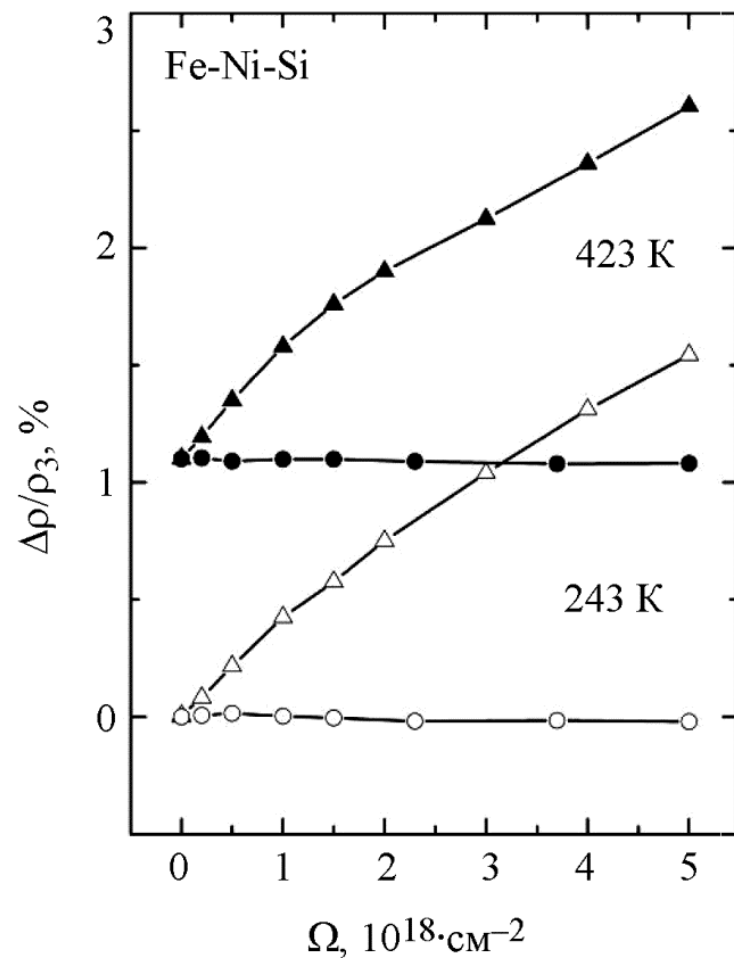

$a$

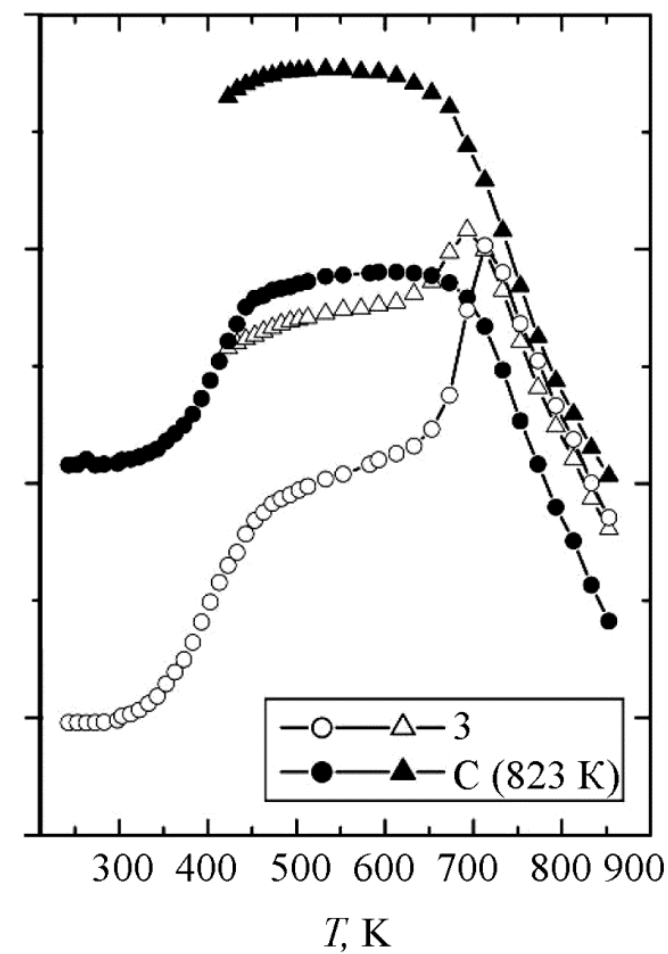

$\sigma$

Рис. 2. Изменения электросопротивления сплава $\mathrm{Fe}-\mathrm{Ni}-\mathrm{Si}$, состаренного (823 K) и закаленного, при облучении электронами при 243 К и 423 К и при изохронном отжиге этих же образцов после облучения

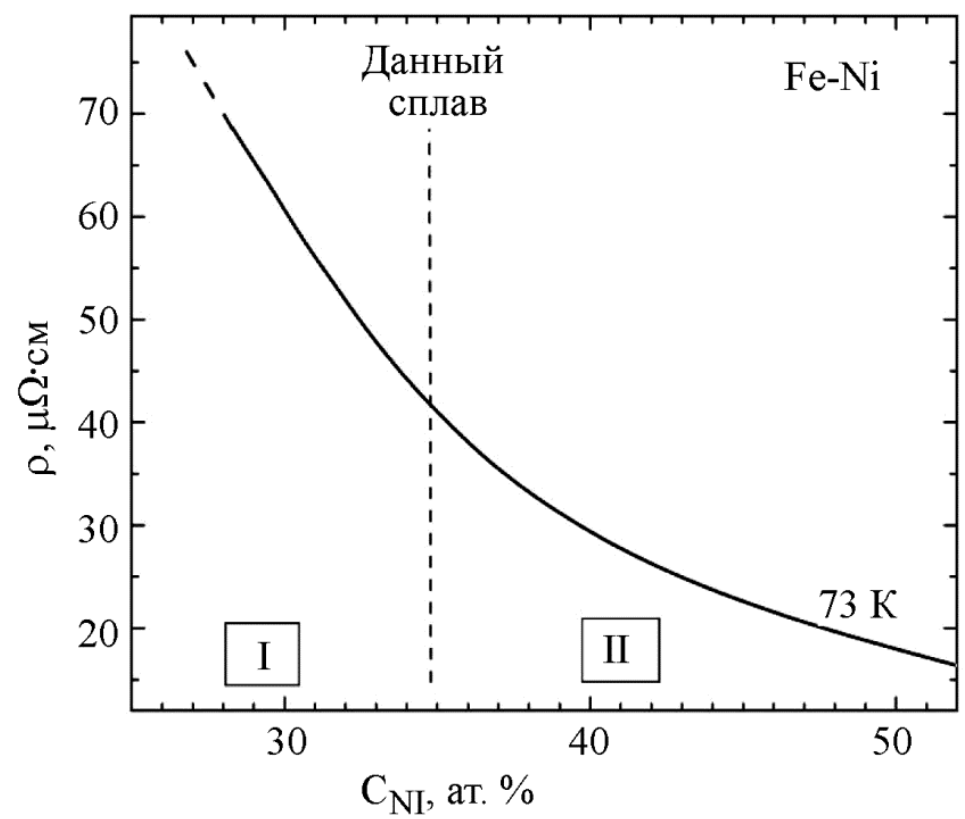

Рис. 3. Зависимость электросопротивления от концентрации никеля в сплаве $\mathrm{Fe}-\mathrm{Ni}$ в области составов, близких к инварному [7]

При дальнейшем повышении температуры в районе 600-900 К, также как и при отжиге состаренных сплавов, происходит термически активируемый распад твердого раствора до 700 К и при более высоких температурах растворение выделений в соответствии с фазовой диаграммой (рис. 1 б). 
При температурах 800 К гомогенизация твердого раствора продолжается на всех сплавах, аналогично сплавам $\mathrm{Fe}-\mathrm{Ni}-\mathrm{Si}$, закаленном и состаренном (823 K) состоянии.

На рис. 3. представлена зависимость электросопротивления сплава $\mathrm{Fe}-\mathrm{Ni}$ от содержания никеля [7]. Электросопротивление повышается с понижением содержания никеля.

При образовании выделений типа $\mathrm{Ni}_{3} \mathrm{Si}$ в сплаве $\mathrm{Fe}-\mathrm{Ni}-\mathrm{Si}$ происходит понижение концентрации никеля в твердом растворе. Предположим аналогичную зависимость электросопротивления от концентрации никеля в сплаве $\mathrm{Fe}-\mathrm{Ni}-\mathrm{Si}$. Можно оценить, какое количество $\mathrm{Ni}$ при этом выводится из твердого раствора. Для получения роста электросопротивления в 1,5 \%, наблюдающегося при облучении сплава $\mathrm{Fe}-\mathrm{Ni}-\mathrm{Si}(423 \mathrm{~K})$, при удельном электросопротивлении сплава около 100 мкОм·см, изменение концентрации $\mathrm{Ni}$ составит около 0,5 ат. \%, а изменение концентрации $\mathrm{Si}$ - около 0,17 ат. \%. Становится понятным, почему при образовании выделений и выводе кремния из твердого раствора, электросопротивление сплава Fe-Ni-Si не снижается, a, наоборот, повышается.

\section{4. Выводы}

Методом остаточного электросопротивления проведены исследования структурнофазовых изменений в инварном сплаве $\mathrm{Fe}-\mathrm{Ni}-\mathrm{Si}$ при термическом старении и электронном облучении.

1. Область заметного влияния термической диффузии в сплавах $\mathrm{Fe}-\mathrm{Ni}-\mathrm{Si}$ начинается выше при температуре $600 \mathrm{~K}$ и обеспечивает распад твердого раствора с повышением остаточного электросопротивления, а выше $700 \mathrm{~K}$ наблюдается гомогенизация с растворением интерметаллидов в соответствии с равновесной фазовой диаграммой.

2. При облучении выше 240 К происходит образование вакансионных кластеров как в состаренном, так и в закаленном сплавеFe-Ni-Si. Диссоциация вакансионных кластеров, приводящая к образованию свободно мигрирующих вакансий, происходит в температурном интервале $350-500 \mathrm{~K}$.

3. При миграции вакансий, генерированных облучением, либо возникающих при диссоциации кластеров, происходит распад твердого раствора в сплаве $\mathrm{Fe}-\mathrm{Ni}-\mathrm{Si}$ с образованием и ростом интерметаллидных выделений.

\section{Благодарность}

Работа выполнена в рамках государственного задания МИНОБРНАУКИ России (тема «Функиия» № 0388-2019-0004).

\section{Литература}

1. Microstructural evolution and radiation stability of steels and alloys / V. N. Voyevodin, I. M. Neklyudov, V. V. Bryk, O. V. Borodin // J. Nucl. Mater. - 1999. - Vol. 271-272. P. 290-295. - DOI: 10.1016/S0022-3115(98)00785-5.

2. Dimitrov C., Dimitrov O. Composition dependence of defect properties in electron irradiated Fe-Cr-Ni solid solutions // J. Phys. F: Metal Phys. - 1984. - Vol. 14. - P. 793-811. DOI: 10.1088/0305-4608/14/4/005.

3. Effect of neutron and electron irradiation on radiation-induced separation of solid solutions in the Fe-Ni and Fe-Ni-P alloys / V. L. Arbuzov, B. N. Goshchitskii, S. E. Danilov, A. V. Kar'kin, D. A. Perminov // The Physics of Metals and Metallography. - 2008. - Vol. 106, No. 3. P. 266-275. - DOI: 10.1134/S0031918X08090068.

4. The influence of intermetallic ageing during irradiation by fast neutrons on void formation in austenitic stainless steels / V. V. Sagaradze, V. A. Pavlov, V. M. Alyabiev, B. N. Goshchitskiy, A. V. Kozlov, S. S. Lapin, Ye. N. Loguntsev, V. M. Nalesnik, N. V. Khakhalkin, V. I. Shalayev, M. G. Gaydukov, G. A. Sergeyev // The Physics of Metals and Metallography. - 1988. - Vol. 65, No. 5. - P. 128-135. 
5. Druzhkov A. P., Perminov D. A., Davletshin A. E. The effect of alloying elements on the vacancy defect evolution in electron-irradiated austenitic $\mathrm{Fe}-\mathrm{Ni}$ alloys studied by positron annihilation // J. Nucl. Mat. - 2009. - 384 (1). - P. 56-60. - DOI: 10.1016/j.jnucmat.2008.10.002.

6. Druzhkov A. P., Arbuzov V. L., and Danilov S. E. The effect of deuterium and tritium on formation and annealing of vacancy-type defects in deformed nickel // Physica Status Solidi (a) Applications and Materials Science. - 2008. - Vol. 205, iss. 7. - P. 1546-1551. DOI: $10.1002 /$ pssa.200723494.

7. Couderchon G., Porteseil J. L. Some properties of nickel-rich commercial Fe-Ni alloys // The Iron-Nickel alloys / ed. by G. Beranger et al. - Lavoisier Publishing. - 1996. - P. 29-58.

8. The influence of plastic deformation and radiation defects on the structural and phase transformations of N36 and N36T2 austenitic alloys / V. L. Arbuzov, S. E. Danilov, A. P. Druzhkov, D. A. Perminov // The Physics of Metals and Metallography. - 2004. - Vol. 98, No. 5. P. 500-505.

9. Takeda Shuzo, Iwama Yoshiroh, Sakakura Akira. On the Equilibrium Diagram of Fe-Ni-Si System and the Ternary Compound y-Phase Appearing in This System (Fundamental Research of Constant Permeability Alloys, 2nd Report) // Journal of the Japan Institute of Metals and Materials. - 1960. - Vol. 24, iss. 8. - P. 534-538. - DOI: 10.2320/jinstmet1952.24.8_534.

10. Алисова С. П., Будберг П. Б., Агеев Н. В. Диаграммы состояния металлических систем. - Москва (Люберцы), ВИНИТИ, 1971. - Вып. 15. - 263 с. 